\title{
USING AUGMENTED REALITY FOR ENTERTAINMENT
}

\author{
Blair MacIntyre and Brendan Hannigan \\ Georgia Institute of Technology
}

\begin{abstract}
In this paper we will discuss the initial results of an ongoing research project exploring the potential of augmented reality as an entertainment medium. We summarize two projects that exploit different aspects of the relationship between the physical and virtual worlds in immersive first person augmented reality (AR), and report on their results. The first project, $A$ Mad Tea Party, explores how procedural characters and symmetries in the physical and virtual worlds can expose interaction possibilities and increase immersion of the experience. The second, Three Angry Men, explores how the first person point-of-view and temporal discontinuities can be used to create a compelling experience. We will also briefly discuss our conceptual framework of remediating existing media forms (film, stage, and interactive CD-ROM) and building on cultural expectations of our users.
\end{abstract}

Key words: augmented reality (AR), remediation, media theory

\section{Introduction}

In this paper, we present initial results of a series of collaborative experiments between researchers in New Media Design and Computing, investigating the creation of engaging, dramatic augmented reality (AR) entertainment experiences. For these projects, we focus on AR techniques that utilize personal displays (such as head-worn displays) that directly augment a person's perception of their surroundings, and localized 3D sounds delivered by personal headphones. Unlike other first person experiences (virtual reality or desktop systems), the augmented reality medium operates within the physical world, from the user's natural point of view. Furthermore, we hope that personal AR techniques can achieve a

The original version of this chapter was revised: The copyright line was incorrect. This has been corrected. The Erratum to this chapter is available at DOI: 10.1007/978-0-387-35660-0_65 
level of immersion beyond that of other mixed reality approaches. Both projects discussed here investigate ways of exploiting the relationship between the physical and virtual worlds in first-person AR to create engaging experiences.

\section{Conceptual Framework}

Our approach to constructing dramatic AR experiences is based on the idea of treating AR as a new media, and leveraging the conceptual framework of new media design $[2,9,12]$. This manifests itself in two ways. First, we recognize that all users will perceive any experience within their cultural context. Therefore, we leverage specific cultural expectations in our designs. For example, the Three Angry Men experience is designed assuming the racial tension of $20^{\text {th }}$ century America [6].

Second, we recognize the all users perceive a new media form by implicitly or explicitly relating it to previous media they have experienced. Since AR is a new technology, we cannot make assumptions that the user has any familiarity with display or interaction techniques used in current systems. However, their experiences from film, stage, TV, and computer games create a starting point for their interpretation and understanding of any AR experience.

Therefore, we remediate established media forms to leverage users expectations based on these previous media experiences. Looking at design methods from film and interactive computer games, for example, have allowed us to create more understandable, enjoyable experiences.

\section{AR Entertainment Experiences}

AR (or MR) entertainment experiences can be defined in a large part by their relationship to the physical world. Such experiences must have more than an incidental, or technological, relationship to the physical world. For example, projecting a simulation game (such as SimCity) onto a tabletop will not add anything to the experience (beyond superficial novelty) unless the game leverages the physical world in some way, such as by providing a physical interface that allows the player to directly interact with the simulated world [1,5]. While physical interaction is one compelling motivation for MR experiences, we are particular excited by experiences that incorporate the physical world into the content of experience, rather than only using the physical world as an interface to an otherwise virtual experience. 
In our recent work, we have been creating AR experiences where virtual characters are integrated into carefully designed physical settings $[3,7,8,10,11]$. In each of the two experiences discussed in this paper, we have explored particular aspects of the relationship between the physical and virtual worlds, and how they can contributed to the design of compelling AR experiences.

\subsection{A Mad Tea Party: "Live Characters" and Symmetry}

One challenge encountered when creating AR entertainment spaces is the development of worlds that "feel" natural and unconstrained. This is especially apparent when designing the behavior of, and interactions with, virtual characters.

The Mad Tea-Party AR experience [7] is based on a chapter from Lewis Carroll's Alice's Adventures in Wonderland. The user plays the role of Alice, who sits at the tea party with three interactive characters: the Mad Hatter, Dormouse, and March Hare. The user's objective is to get directions to "the garden" from one of the other partygoers. We add to the sense of reality in the world by having the characters interact with the user and with each other. Each character is procedurally driven, continually engaging the other characters by performing actions such as serving tea, receiving tea, sipping tea, asking riddles, etc. The characters also react to each other, and the user, including reacting to activity not directed at them (such as one character laughing when the user splashes another character with tea).

Procedural characters such as these are often used in modern games, but are especially important in AR experiences because poorly designed characters will stand out in stark contrast to the physical world. Furthermore, it is important that the relationship between the characters and the physical world is also believable. In the Mad Tea Party, we attempt to create the illusion of a "real" world by having the experience progress with or without the user. We found that if virtual characters are only reactive to user stimulus, the experience can stall (relative to the physical world) while waiting for user input, breaking the user's sense of immersion. To avoid this effect, the characters in Alice are each given their own objectives, which they will pursue regardless of user action. The Mad Hatter wants to tell riddles, the March Hare to serve tea, and the Dormouse to sleep. Throughout the experience, the independent behaviors of the characters add to the feeling of believability of the story world and the virtual characters themselves. 
The second technique we explored with the Mad Tea Party was the use of symmetry between the physical and virtual worlds to subtly lead the user toward valid interactions with the virtual characters. We used symmetry in the content of the world (the characters have virtual teapots and teacups, the user has a similar physical tea pot), as well as symmetry of action (the actions that the characters engage in are all valid user actions). By observing the activity of the virtual characters, the majority of users of our system attempted valid actions with no instruction on the use of the system.

Of course, these techniques do not solve the unattainable problem of creating artificially intelligent characters than can understand and interact naturally with the user. However, they do highlight that careful design of the physical and virtual environments can create engaging experiences, even in the absence of truly intelligent characters.

\subsection{Three Angry Men: Point of View and Temporal Discontinuity}

In the Mad Tea Party, we explored techniques for creating an engaging AR experience by blending the virtual world as tightly as possible into the real world. However, the converse (breaking links with the physical world) can also be used to create engaging and dramatic experiences. In the Three Angry Men project, we explored how changes in point of view and breaking temporal synchronization between the physical and virtual world can be used effectively.

Since AR applications using personal displays are naturally viewed in the first person, a change in the point of view can be used for a number of dramatic effects. In Three Angry Men, first person perspective affords us the ability to change the identity of the user with a change in view, and gives them a sense of being somewhere between the first and third person perspective on the scene. The Three Angry Men experience is based on the Reginald Rose 1954 play "12 Angry Men" (later made into movies in '57 and '97), where 12 jurors sit and decide on the fate of a young man accused of murder. In Three Angry Men, the user assumes the role of one of three jurors deliberating in a jury room (the experience was designed for 3 instead of 12 to ease technological constraints), and participates in a 6-minute scene that was assembled from the original script.

Three Angry Men takes place at a table, where the user sits and participates in a scene with two other jurors, portrayed using video actors [8]. While playing the role of a particular juror, the user will experience the scene from his perspective. 


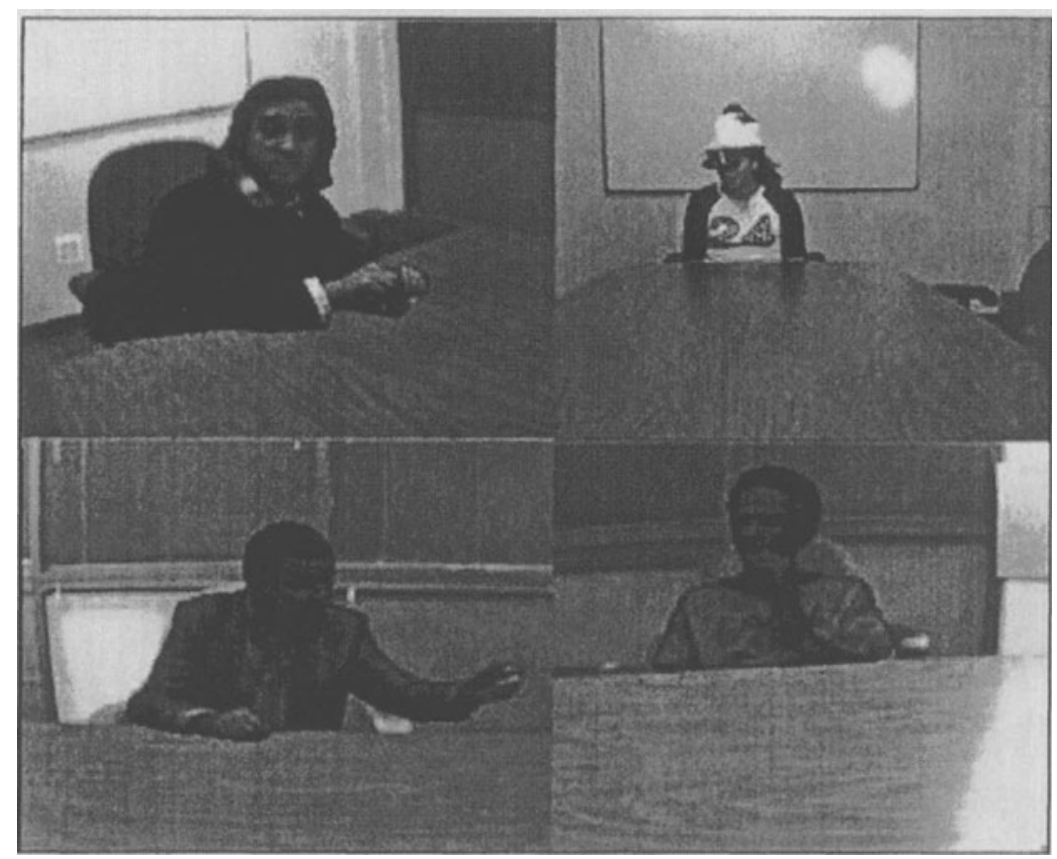

Figure 1. the user assumes the role of one of three jurors

At any time, however, the user can stand from the table, stepping out of the body of their character and causing the unfolding scene to stop. When the user sits in a different juror's chair, the scene is continued from the new character's perspective. Different jurors will see the same scene acted very differently, with the other virtual actors changing their posture, clothes, grooming, and the way they deliver their lines to accentuate the differences in personal biases of the user's current persona. The disconnection of the physical and virtual world breaks the users sense of identity with the most recent character, causing an experience more akin to a ghost inhabiting a person's body than the illusion of being that character. 


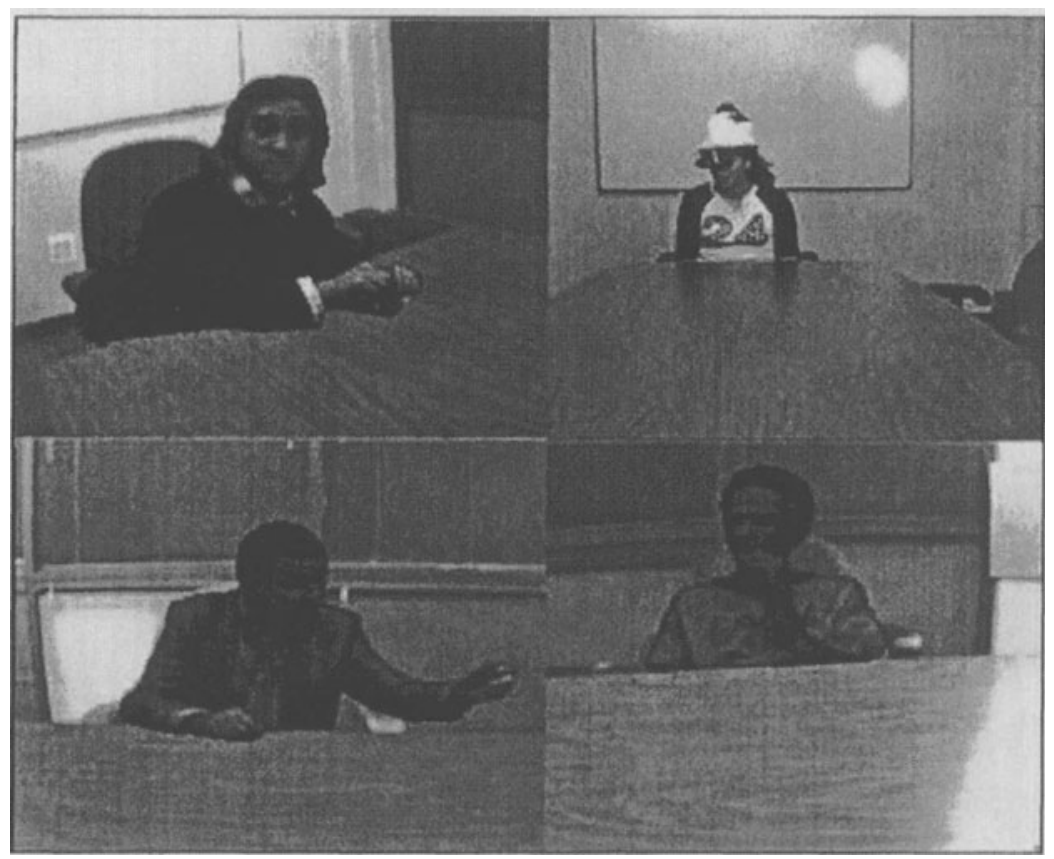

Figure 2. users see the biases of the jurors as they change positions

We also use temporal breaks between the physical and virtual world to mimic the soliloquies of live theatre [4]; stage plays employ stops in action to focus audience attention on an actor as they "step out" of the action and convey their inner thoughts. We employ the theatrical soliloquy paradigm to allow the inner thoughts of the currently occupied character to be conveyed to the user. In Three Angry Men, we pause the scene and fade the graphics while the user experiences his or her character's thoughts on the deliberation, revealing each character's biases and prejudices. The experience allows us to see into the minds of its characters, without interfering with the dialogue of the scene.

\section{Conclusions}

We have presented two dramatic $\mathrm{AR}$ experiences, and discussed the techniques we used to exploit the unique characteristics of $A R$ to enhance the overall entertainment experience. We have found that a key feature of $\mathrm{AR}$ experiences is that they immerse users in the scene, instead of simply presenting a scene to them through a personal display. Allowing people to interact in a world with independent, physically located characters brings 
them into the story, and this sense of participation proves to be a valuable tool in dramatic design.

We have also experimented with changing the persona of the user using switches in point of view and temporal discontinuities, creating a unique mix of first and third person experiences. We are currently exploring other techniques for leveraging the juxtaposition of the physical and virtual worlds, by designing with explicit attention to the similarities and differences of these worlds.

\section{References}

[1] Billinghurst, M., Kato, H., and Poupyrev, I. (2001) "The MagicBook-Moving seamlessly between reality and virtuality." IEEE Computer Graphics and Applications, 21(3): pp. 6-8.

[2] Bolter, J.D., and Grusin, R. (1999) Remediation: Understanding New Media. Cambridge MA, MIT Press.

[3] Bolter, J.D., MacIntyre, B., Philip., K., and Harpold, T. (2000) "Cultural Narrative in Augmented Reality." Presented at Digital Arts and Culture (DAC) 2000 , Bergen, Norway. August 2-4, 2000.

[4] Craven, Mike, Taylor, I., Drozd, A., Purbrick, J., Greenhalgh, C., Benford, S., Fraser, M., Bowers, J., Jää- Aro, K., Lintermann, B., and Hoch, M. (2001) "Exploiting Interactivity, Influence, Space and Time to Explore non-Linear Drama in Virtual Worlds." In Proc. ACM CHI 2001, Seattle, WA, March 31-April 5.

[5] Curtis, D., Mizell, D., Gruenbaum, P., and Janin, A. (1998) "Several Devils in the Details: Making AR App Work in the Airplane Factory." In Proc. IWAR '98, pp.47-60.

[6] MacIntyre, B. Bolter, J., Vaughan, J., Hannigan, B., Moreno, E., Haas, M., Gandy, M. (2002) "Three Angry Men: Dramatizing Point-of-View using Augmented Reality". In Proc. SIGGRAPH 02, to be published.

[7] MacIntyre, B. Bolter, J., Moreno, E., Hannigan, B. (2001) "Augmented Reality as a New Media Experience”. In Proc. International Symposium on Augmented Reality (ISAR) '01, pp. 197-206.

[8] MacIntyre, B., Lohse, M., Bolter, J.D., and Moreno, E. (2001) "Ghosts in the Machine: Integrating 2D Video Actors into a 3D AR System." In International Symposium on Mixed Reality 2001, Yokohama, Japan, March 14-15.

[9] Manovich, Lev. (2001) The Language of New Media. Cambridge MA: MIT Press, to be published.

[10] Moreno, E. (2001) “Alice's Adventures in New Media: Towards a Collaborative Language for Augmented Reality." MA Thesis in Information Design and Technology, School of Literature, Communication and Culture, Georgia Tech.

[11] Moreno, E., MacIntyre, B. and Bolter, J.D. (2001) “Alice's Adventure's in New Media: An Exploration of Interactive Narratives in Augmented Reality." To appear in CAST'01, Bonn, Germany, October 21-22.

[12] Oren, T. (1990). "Designing a new medium." In B. Laurel (Ed.), The Art of HumanComputer Interface Design (pp. 467-479). Reading, Mass.: Addison-Wesley. 